\title{
Keep posting
}

\section{During its 25 years of existence, arXiv has exceeded every expectation in terms of growth and its impact on how science is disseminated.}

Things rarely go as planned. Such was the case for an electronic bulletin board dedicated to a small community of theoretical high-energy physicists, which received its first submission on 14 August 1991. Expectations were modest: one hundred articles a year, each to be stored for just three months. But hep-th@xxx.lanl.gov, now known as arXiv.org, has exceeded all expectations. No article has ever been deleted - the total count is now over 1.16 million (Fig. 1), with 110,000 new submissions expected in 2016. With more than 1.2 million access requests per day, arXiv is central to how modernday mathematics, physics and, increasingly, other disciplines function.

ArXiv came about at a time when personal computers were something of a luxury, even for universities, and the Internet was essentially a playground for academics. In 1991, Paul Ginsparg, then a physicist at the Los Alamos National Laboratory, decided to automate the manual task of collating and distributing recent relevant physics articles to a mailing list. As he put it, "it was supposed to be a three-hour tour, not a life sentence" (Nature 476, 145-147; 2011). The original software he wrote was an e-mail transponder written in CSH. A couple of years later, it simply transitioned to the

a

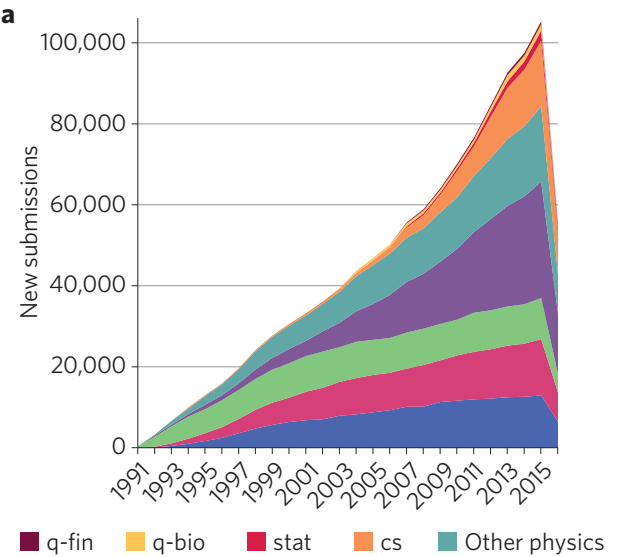

World Wide Web. That was before any of the established physics journals had started adopting electronic technologies for their submission systems. Many did not even have a website - www.nature.com first went live in 1996. ArXiv has influenced the way that scientific journals present themselves online. And its simplicity and efficiency is probably what made it so popular and reliable in the first place, and what makes its users today wary of significantly changing it (http://go.nature.com/29GCztv).

With arXiv gaining popularity, people began to question the need for traditional physics journals. Back in 1994, Ginsparg expressed his doubts about the future of publishing: "in the long term, however, it is difficult to imagine how the current model of funding publishing companies through research libraries (in turn funded by the overhead on research grants) can possibly persist" (http://bit.ly/2a1KUaj). And Ginsparg's opinion remains unchanged, as he shares his views on the present and future of arXiv on page 722 .

Still, 25 years on, subscription-based journals (of which Nature Physics is an example, of course) continue to exist. And despite the existence of freely accessible preprints, open-access journals are on the rise. For now, it seems that journals

b

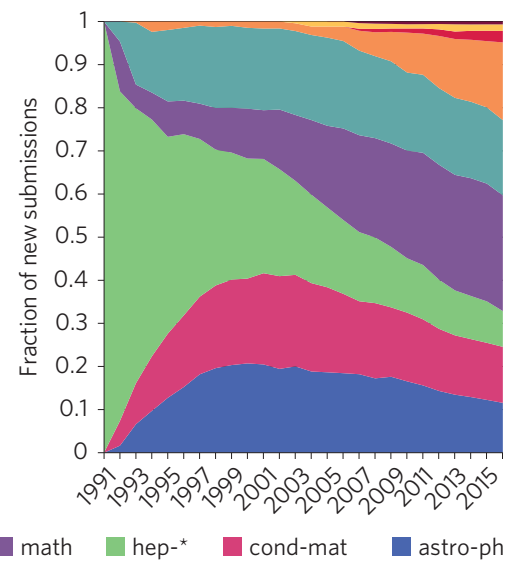

Figure 1 | 25 years of arXiv. $\mathbf{a}, \mathbf{b}$, The well-known physics repository has been growing (a) and diversifying (b) in terms of content. The plot labels use the standard arXiv classification: q-fin (quantitative finance); q-bio (quantitative biology); stat (statistics); cs (computer science); math (mathematics and mathematical physics); hep (high energy physics, with all of its sub-categories indicated by the asterisk); cond-mat (condensed matter physics); and astro-ph (astrophysics). 'Other physics' includes several categories: general physics, nuclear, general relativity and quantum cosmology, quantum physics and nonlinear sciences. Data courtesy of P. Ginsparg.

\section{Five things you (probably) did not know about arXiv.}

(1) The $\mathrm{X}$ in arXiv stands for the Greek letter chi in TeX and for the $\mathrm{xxx}$ in xxx.lanl.gov. The xxx was used by Paul Ginsparg in TeX files to mark text awaiting corrections (preprint at http:// arXiv.org/abs/1108.2700v2; 2011).

(2) There are 158 moderators who work voluntarily to ensure that all submissions are scientific, relevant to their research community and appropriately classified.

(3) ArXiv does not come cheap. The maintenance costs rose to a bit over US\$800,000 in 2016, and are covered by the Cornell University Library, the Simons Foundation and a collective of institutions worldwide.

(4) There are over 210,000 active submitters, a number that is growing by about $10 \%$ each year.

(5) The proof of the Poincaré conjecture, for which Grigori Perelman was awarded both the Fields Medal and the Millennium Prize, was published exclusively on arXiv.

and arXiv can peacefully coexist. In some cases, they even complement each other: manuscripts are being posted and feedback from the community being received before submission to a journal; longer versions of the papers with additional information are made available on the preprint server; and results are already being disseminated while the peer review proceeds at its deliberate and thorough pace.

Despite a sadly persistent misconception, we at the Nature Research Journals love arXiv. We encourage authors to post their manuscripts before formal submission and update the final version six months after publication. A significant number of the papers have we published are available on arXiv. And when it comes to our vision of the future, we not only imagine coexisting with arXiv, but also hope for better integration - perhaps, one day, our online submission system would have evolved enough to allow authors to submit a paper by simply entering its arXiv number! 